Journal of Applied AnALysis

Vol. 13, No. 1 (2007), pp. 33-56

\title{
RANDOM PARTITION AND STOCHASTIC INTEGRATION IN FINITE VON NEUMANN ALGEBRAS
}

\author{
A. A. A. MOHAMMED \\ Received December 10, 2000 and, in revised form, September 27, 2005
}

\begin{abstract}
We introduce the notion of a random partition of the stochastic interval $\left[\tau_{0}, \tau_{\infty}\right]$ as an analogy to the classical case and characterize the predictable processes associated with such partitions. Also we identify the operator algebras connected with the stochastic integrals of predictable processes and examine their mutual relations.
\end{abstract}

\section{INTRODUCTION}

A noncommutative stochastic integration for adapted processes with respect to a bounded $L^{2}$-martingale was studied in greater detail in [10] with the idea of viewing the stochastic integral, with fixed integrand and varying integrator, as a bounded linear operator on the Hilbert space of integrators. In connection with these operators various operator algebras arise in a natural way. Our aim in this paper is to introduce the notion of a random partition of the stochastic interval $\left[\tau_{0}, \tau_{\infty}\right]$ (where $\tau_{0}$ and $\tau_{\infty}$ are the smallest and the largest random times, respectively) and use these partitions to

2000 Mathematics Subject Classification. Primary 64L53.

Key words and phrases. Noncommutative stochastic integration, predictable processes, random times, time projections, martingales, von Neumann algebras.

ISSN 1425-6908 (C) Heldermann Verlag. 
identify the operator algebras connected with the stochastic integrals of predictable processes and to examine their mutual relations. The contents of the paper is as follows. In Section 1 we introduce the basic terminology and describe the setup we shall be working in. Section 2 contains a brief review of the notions of predictable processes, random time and the associated time projection. Section 3 contains a brief review of the stochastic integration of adapted processes with respect to a bounded $L^{2}$-martingale, the classes of integrable processes and the operator algebras connected with the stochastic integrals of these classes. In Section 4 we define a random partition of the stochastic interval $\left[\tau_{0}, \tau_{\infty}\right]$ and characterize predictable processes associated with these random partitions (see Theorem 4.2 and Theorem 4.5). Section 5 is devoted to various aspects of random partitions and the related operator algebras. In particular, the operator algebras connected with the stochastic integrals of predictable processes are identified and their mutual relations are examined (see Lemma 5.4, Theorem 5.6 and Proposition 5.8). Finally, in Section 6 we show the operator algebra connected with the stochastic integrals of predictable processes associated with certain random partition is an image of a $*$-preserving strongly continuous positive linear map defined on the product von Neumann algebra of the filtration under consideration (see Lemma 6.4).

\section{Mathematical PRELiminaries}

A noncommutative stochastic base which we shall be working in consists of the following elements: a von Neumann algebra $\mathcal{A}$ acting on a Hilbert space $\mathcal{H}$, a normal faithful tracial state $\varphi$ on $\mathcal{A}$, a filtration $\left(\mathcal{A}_{t}\right), t \in[0,+\infty]$, which is an increasing ( $s \leq t$ implies $\mathcal{A}_{s} \subset \mathcal{A}_{t}$ ) family of von Neumann subalgebras of $\mathcal{A}$ such that $\mathcal{A}=\mathcal{A}_{\infty}=\left(\bigcup_{t \geqslant 0} \mathcal{A}_{t}\right)^{\prime \prime}$ and $\mathcal{A}_{s}=\bigcap_{t>s} \mathcal{A}_{t}$ (right-continuity). Then for each $t \geq 0$ there exists a normal conditional expectation $\mathcal{M}_{t}$ from $\mathcal{A}$ onto $\mathcal{A}_{t}$ such that $\varphi \circ \mathcal{M}_{t}=\varphi$.

For each $t \in[0,+\infty]$ we write $L^{2}\left(\mathcal{A}_{t}\right)$ for the noncommutative Lebesgue space associated with $\mathcal{A}_{t}$ and $\varphi$. The theory of these spaces is described e.g. in [11], for our purpose we recall only that $L^{2}(\mathcal{A})$ (accordingly $L^{2}\left(\mathcal{A}_{t}\right)$ ) consists of densely defined operators on $\mathcal{H}$, affiliated to $\mathcal{A}$, and that $L^{2}(\mathcal{A})$ is the completion of $\mathcal{A}$ with respect to the norm

$$
\|X\|_{2}=\left[\varphi\left(|X|^{2}\right)\right]^{1 / 2}
$$

moreover, for $a \in \mathcal{A}$ and $X \in L^{2}(\mathcal{A})$, the operators $a X$ and $X a$ belong to $L^{2}(\mathcal{A})$. For each $t$ the conditional expectation $\mathcal{M}_{t}$ extends to the projection from $L^{2}(\mathcal{A})$ onto $L^{2}\left(\mathcal{A}_{t}\right)$. 
By an $\mathcal{A}$-valued (respectively $L^{2}$-valued) process we mean a map from $[0,+\infty]$ into $\mathcal{A}$ (respectively $L^{2}(\mathcal{A})$ ). $\mathcal{A}$-valued processes will be usually denoted by $f, g$ or $\left(f_{t}\right),\left(g_{t}\right)$ while the $L^{2}$-processes we shall use the symbols $\left(X_{t}\right),\left(Y_{t}\right)$. An $\mathcal{A}$-valued (respectively $L^{2}$-valued) process $f$ (respectively $X$ ) is called adapted if $f_{t} \in \mathcal{A}_{t}$ (respectively $X_{t} \in L^{2}\left(\mathcal{A}_{t}\right)$ ).

Let $f=\left(f_{t}\right)$ be an arbitrary $\mathcal{A}$-valued adapted process. By $f^{*}$ we denote the adjoint process given by $\left(f_{t}^{*}\right)$. Recall that $\varphi$ is a trace and $L^{2}(\mathcal{A})$ is a Hilbert space with inner product given by

$$
\langle X, Y\rangle=\varphi\left(Y^{*} X\right), \quad \text { for } X, Y \in L^{2}(\mathcal{A}) .
$$

The conditional expectation $\mathcal{M}_{t}$ enjoys the property $\left(\mathcal{M}_{t} f_{t}\right)^{*}=\mathcal{M}_{t}\left(f_{t}^{*}\right)$ $\forall t \in[0,+\infty]$.

An $L^{2}$-process $\left(X_{t}: t \in[0,+\infty)\right)$ is called a martingale if for each $s, t \in$ $[0,+\infty), s \leq t$ we have $\mathcal{M}_{s}\left(X_{t}\right)=X_{s}$. An $L^{2}$-martingale $\left(X_{t}: t \in[0,+\infty)\right)$ is bounded if $\left\|X_{t}\right\| \leq c$ for all $t \geq 0$. An elementary Hilbert space type reasoning shows that boundedness is equivalent to the existence of an element $X_{\infty} \in L^{2}(\mathcal{A})$ such that $X_{t}=\mathcal{M}_{t}\left(X_{\infty}\right), t \geq 0$ (cf. e.g. [3, Proposition 1.1]). Now we can extend $\left(X_{t}\right)$ to the interval $[0,+\infty]$ and $\left(X_{t}: t \in[0,+\infty]\right)$ is clearly a martingale. This means that while speaking about bounded martingales it is inessential whether we consider them for $t \in[0,+\infty]$ or in $[0,+\infty)$.

By a slight abuse of notation we shall drop the subscript " $\infty$ " and write simply $X_{t}=\mathcal{M}_{t}(X)$. Let us adopt the following notational convention. For each $X \in L^{2}(\mathcal{A})$ we denote by $\left(X_{t}\right)$ the martingale given by

$$
X_{t}=\mathcal{M}_{t}(X), \quad t \in[0,+\infty] .
$$

Let us notice that according to [8] any $L^{2}$-martingale adapted to a right continuous filtration of algebras is right $L^{2}$-continuous, so $\mathcal{M}_{t} \longrightarrow \mathcal{M}_{s}$ as $t \searrow s$.

\section{RANDOM TIMES, TIME PROJECTIONS AND PREDICTABLE PROCESSES}

Firstly, we recall the definition of a random time and the associated time projection. The motivation for these definitions and all the details can be found in [4], [5], [7], [8].

Definition 2.1. A random time, $\tau$, is an increasing family of projections $\left(E_{t}\right), t \in[0,+\infty]$ with $E_{t} \in \mathcal{A}_{t}$ and $E_{0}=0, E_{+\infty}=\mathbf{1}$.

Each deterministic time, $t \in[0,+\infty)$, is identified with a random time $\widetilde{t}=\left(E_{s}\right)$ defined by

$$
E_{s}= \begin{cases}0, & s \leq t \\ 1, & s>t\end{cases}
$$


When $t=+\infty$, it is identified with a random time $\widetilde{\infty}=\left(F_{t}\right)$ defined by

$$
F_{s}= \begin{cases}0, & s<+\infty \\ \mathbf{1}, & s=+\infty\end{cases}
$$

\section{Definition 2.2.}

1. For two random times $\tau=\left(E_{t}\right)$ and $\sigma=\left(F_{t}\right)$ we say that $\tau \leq \sigma$ if $F_{t} \leq E_{t}, \forall t \in[0,+\infty]$. We define $\tau \wedge \sigma$ and $\tau \vee \sigma$ to be the random times $\tau \wedge \sigma=\left(E_{t} \vee F_{t}\right)$ and $\tau \vee \sigma=\left(E_{t} \wedge F_{t}\right)$.

2. For $\Theta$ the set of all finite partitions of $[0,+\infty]$ and $\theta=\left\{0=t_{0}<t_{1}<\right.$ $\left.\ldots<t_{n}=+\infty\right\} \in \Theta$, we define an operator $\mathcal{M}_{\tau(\theta)}$ on $L^{2}(\mathcal{A})$ by the formula

$$
\mathcal{M}_{\tau(\theta)}(X)=\sum_{i=1}^{n} \mathcal{M}_{t_{i}}(X)\left(E_{t_{i}}-E_{t_{i-1}}\right), \quad X \in L^{2}(\mathcal{A}) .
$$

$\mathcal{M}_{\tau(\theta)}$ has the following properties (see [5, Theorem 2.3]):

1. $\mathcal{M}_{\tau(\theta)}$ is an orthogonal projection;

2. For $\theta, \eta \in \Theta$ with $\eta$ finer than $\theta, \mathcal{M}_{\tau(\eta)} \leq \mathcal{M}_{\tau(\theta)}$;

3. If $\sigma$ is another random time with $\tau \leq \sigma$ then $\mathcal{M}_{\tau(\theta)} \leq \mathcal{M}_{\sigma(\theta)}, \forall \theta \in \Theta$.

In view of these properties and the fact that $\Theta$ is a directed set ordered by inclusion we note that $\left\{\mathcal{M}_{\tau(\theta)}: \theta \in \Theta\right\}$ becomes a decreasing net of orthogonal projections. Hence there exists a unique orthogonal projection

$$
\mathcal{M}_{\tau}=\lim _{\theta} \mathcal{M}_{\tau(\theta)}=\bigwedge_{\theta \in \Theta} \mathcal{M}_{\tau(\theta)}
$$

in the strong operator topology as $\theta$ refines. We shall call $\mathcal{M}_{\tau}$ the time projection for the random time $\tau$ ([5, Definition 2.4]). As an immediate corollary of [7], we have the Optional Stopping Theorem which states that for random times $\tau, \sigma$ with $\tau \leq \sigma$, we have $\mathcal{M}_{\tau} \leq \mathcal{M}_{\sigma}$.

It is a simple matter to verify that the time projection associated with a random time $\widetilde{t}$ agrees with $\mathcal{M}_{t}$, the conditional expectation. Consequently, $\mathcal{M}_{\widetilde{0}}=\mathcal{M}_{0}$ is the smallest time projection while $\mathcal{M}_{\widehat{\infty}}=\mathbf{1}$ is the largest time projection.

Let us recall the following definition from [8], [10].

\section{Definition 2.3.}

1. Let $\sigma=\left(F_{t}\right)$ and $\tau=\left(E_{t}\right)$ be random times with $\sigma \leq \tau$. The stochastic interval $(\sigma, \tau]$ is a process defined as

$$
(\sigma, \tau](t)=F_{t}-E_{t}, \quad t \in[0,+\infty] .
$$


2. For a projection $P$ in $\mathcal{A}_{0}$ we define "an interval" $\left[0_{P}\right]$ as

$$
\left[0_{P}\right](t)= \begin{cases}P & \text { for } t=0 \\ 0 & \text { otherwise }\end{cases}
$$

3. Let $\lambda_{i}$ for $i=0,1, \ldots, n$, be complex numbers, $P$ a projection in $\mathcal{A}_{0}$, and $\sigma_{1}, \ldots, \sigma_{n}, \tau_{1}, \ldots, \tau_{n}$ random times with $\sigma_{1} \leq \tau_{1} \leq \sigma_{2} \leq \tau_{2} \leq$ $\ldots \leq \sigma_{n} \leq \tau_{n}$. Any process $f$ of the form

$$
f=\lambda_{0}\left[0_{P}\right]+\sum_{i=1}^{n} \lambda_{i}\left(\sigma_{i}, \tau_{i}\right]
$$

is called an elementary predictable process. The class of all elementary predictable processes is denoted by $\mathcal{E}$.

4. A process which is a finite linear combination of finite products of elementary predictable processes is called a simple predictable process. The class of all simple predictable processes is denoted by $\mathfrak{S}$.

5. A process $f=\left(f_{t}\right)$ is called a bounded predictable process if there is a sequence, $f^{n}=\left(f_{t}^{n}\right)$, of simple predictable processes, converging in operator norm pointwise to $f$ and which satisfy

$$
\sup _{n} \sup _{t}\left\|f_{t}^{n}\right\|_{\infty}<+\infty
$$

The class of all bounded predictable processes is denoted by $\mathcal{P}$. Note first of all that every bounded predictable process takes the value 0 at $t=+\infty$.

In [10] an important class of predictable processes has been introduced, called uniformly predictable and defined as follows:

Definition 2.4. A process $f$ is called uniformly predictable if it is the uniform limit of a sequence of simple predictable processes. The class of all uniformly predictable processes is denoted by $\mathcal{U}$.

Thus $f$ is uniformly predictable if there exists a sequence, $f_{n}=\left(f_{t}^{(n)}\right)$, of simple predictable processes and

$$
\lim _{n \rightarrow \infty} \sup _{t}\left\|f_{t}^{(n)}-f_{t}\right\|_{\infty}=0 .
$$

\section{Stochastic InTEGRATION FOR ADAPTED PROCESSES}

We recall the definition and some properties of stochastic integration with respect to a bounded $L^{2}$-martingale processes. We also recall the definitions of various classes of integrable processes and the operator algebras connected with stochastic integrals of these classes. For details and proofs the reader is referred to [10]. 
Let $f$ be an $\mathcal{A}$-valued process and $\left(X_{t}\right)$ an $L^{2}(\mathcal{A})$-valued process. For a partition $\theta=\left\{0=t_{0}<t_{1}<\ldots<t_{m}=+\infty\right\}$ of $[0,+\infty]$ we form integral sums

$$
\begin{aligned}
& S_{\theta}^{l}(f, X)=\sum_{i=1}^{m}\left(X_{t_{i}}-X_{t_{i-1}}\right) f_{t_{i-1}}, \\
& S_{\theta}^{r}(f, X)=\sum_{i=1}^{m} f_{t_{i-1}}\left(X_{t_{i}}-X_{t_{i-1}}\right),
\end{aligned}
$$

call the left and the right sum, respectively. Both the sums are elements of $L^{2}(\mathcal{A})$, and if there exist $L^{2}$-limits of these sums as $\theta$ refines, they are called the left and right integral of $f$ with respect to $\left(X_{t}\right)$, respectively, and are denoted by

$$
\begin{aligned}
\lim _{\theta} S_{\theta}^{l}(f, X) & =\int d X_{t} f_{t} \\
\lim _{\theta} S_{\theta}^{r}(f, X) & =\int f_{t} d X_{t} .
\end{aligned}
$$

The various classes of integrable processes are introduced by the following definition.

Definition 3.1. Let $f$ be an $\mathcal{A}$-valued uniformly bounded adapted process.

1. $f$ is called completly left integrable if $\int d X_{t} f_{t}$ exists for each $X \in$ $L^{2}(\mathcal{A})$. The class of all completely left integrable process is denoted by $\mathcal{L}$.

2. $f$ is called completly right integrable if $\int f_{t} d X_{t}$ exists for each $X \in$ $L^{2}(\mathcal{A})$. The class of all completely right integrable process is denoted by $\mathcal{R}$.

3. $f$ is called completly integrable if it is completely left and right integrable. The class of all completely integrable processes is denoted by $\mathcal{I}$. Thus

$$
\mathcal{I}=\mathcal{L} \cap \mathcal{R}
$$

As for this theory of integration, it turns out that the class $\mathcal{U}$ of uniformly predictable processes is fundamental; i.e. the uniformly predictable processes are completely left and right integrable processes, see Proposition 2.1.3 and Corollary 2.1.8 of [10]. Consequently, $\mathcal{E} \subset \mathfrak{S} \subset \mathcal{U} \subset \mathcal{I}$.

For an $\mathcal{A}$-valued uniformly bounded process $f$ put

$$
\|f\|=\sup _{t}\left\|f_{t}\right\|_{\infty} .
$$

For each $f \in \mathcal{L}, L_{f}$ is a bounded linear operator on $L^{2}(\mathcal{A})$ defined by

$$
L_{f}(X)=\int d X_{t} f_{t}, \quad X \in L^{2}(\mathcal{A}),
$$


and $\left\|L_{f}\right\| \leq\|f\|$. Similarly, for each $f \in \mathcal{R}, R_{f}$ is a bounded linear operator defined by

and $\left\|R_{f}\right\| \leq\|f\|$.

$$
R_{f}(X)=\int f_{t} d X_{t}, \quad X \in L^{2}(\mathcal{A})
$$

Theorem 3.2 ([10, Theorem 2.1.5]).

1. $\mathcal{L}$ with norm $\|\cdot\|$ is a Banach algebra, and the map $\mathcal{L} \ni f \longrightarrow L_{f}$ is an antirepresentation $\left(L_{f g}=L_{g} L_{f}\right)$ of $\mathcal{L}$ in $B\left(L^{2}(\mathcal{A})\right)$.

2. $\mathcal{R}$ with norm $\|\cdot\|$ is a Banach algebra, and the map $\mathcal{R} \ni f \longrightarrow R_{f}$ is a representation of $\mathcal{R}$ in $B\left(L^{2}(\mathcal{A})\right)$.

3. I with norm $\|\cdot\|$ is a $C^{*}$-algebra. The map $\mathcal{I} \ni f \longrightarrow R_{f}$ is $a^{*}$ representation and the map $\mathcal{I} \ni f \longrightarrow L_{f}$ is $a^{*}$-antirepresentation of $\mathcal{I}$ in $B\left(L^{2}(\mathcal{A})\right)$.

In what follows we formulate our definition and result in the "left" versions, their "right" counterparts are formulated in an obvious manner and have virtually the same proofs as the left ones. In connection with these operators various operator algebras arise in a natural way. Let us recall the following definition from [10].

\section{Definition 3.3.}

1. By $\mathcal{I}_{l}(\mathcal{A})$ we denote the von Neumann algebra on $L^{2}(\mathcal{A})$ generated by all operators $L_{f}$, where $f$ is a completely integrable process, i.e.

$$
\mathcal{I}_{l}(\mathcal{A})=\left\{L_{f}: f-\text { a completly integrable process }\right\}^{\prime \prime} .
$$

2. By $\mathcal{L}(\mathcal{A})$ we denote the von Neumann algebra on $L^{2}(\mathcal{A})$ generated by all operators $L_{f}$, where $f$ is a completely left integrable process, i.e.

$$
\mathcal{L}(\mathcal{A})=W^{*}\left(\left\{L_{f}: f-\text { a completly left integrable process }\right\}\right) .
$$

Now, take the constant process equal to 1 - the identity of the algebra $\mathcal{L}$ we immediately get $L_{1}=\mathcal{M}_{0}^{\perp}$, which means that the algebra $\left\{L_{f}: f \in \mathcal{L}\right\}$ acts nondegenerately on the subspace $\mathcal{M}_{0}^{\perp}\left(L^{2}(\mathcal{A})\right.$ ) of $L^{2}(\mathcal{A})$ (and is zero on $\mathcal{M}_{0}\left(L^{2}(\mathcal{A})\right)$ ). If we wanted to explicitly take this fact into account we could consider e.g. the algebra

$$
W^{*}\left(\left\{\lambda_{0} \mathcal{M}_{0}+L_{f}: f \in \mathcal{L}, \lambda_{0} \in \mathbb{C}\right\}\right) .
$$

The above mentioned "deficiency" of the operators $L_{f}$ is probably the reason for their slightly different definition in [8] (see [8, Definition 5.3]) namely they are defined as

$$
\widetilde{L}_{f}=L_{f}+\mathcal{M}_{0}(\cdot) f_{0} .
$$

In [10] it was shown that there is an interesting and naturally looking setup to which the above described operators $\widetilde{L}_{f}$ fit well. Indeed, by can 
use the generalization of a random time which is called quantum stopping time, by which we mean a non-decreasing family of projections $\tau^{\prime}=\left(E_{t}^{\prime}\right)$ such that $E_{t}^{\prime} \in \mathcal{A}_{t}$ for each $t \in[0,+\infty)$ and $E_{\infty}^{\prime}=1$ (cf. [3, Definition 1.1]). In this work we shall consider the definition $\widetilde{L}_{f}$ above and restrict our attention to the various classes of predictable processes. Let us consider the following definition which is adopted from [10] and [8].

\section{Definition 3.4.}

1. By $\widetilde{\mathcal{E}(\mathcal{A})}$ we denote the von Neumann algebra on $L^{2}(\mathcal{A})$ generated by all the operators $\widetilde{L}_{f}$, where $f$ is an elementary predictable process, i.e.

$$
\widetilde{\mathcal{E}(\mathcal{A})}=\left\{\widetilde{L}_{f}: f-\text { an elementary predictable process }\right\}^{\prime \prime} .
$$

2. By $\widetilde{\mathcal{U}(\mathcal{A})}$ we denote the von Neumann algebra on $L^{2}(\mathcal{A})$ generated by all the operators $\widetilde{L}_{f}$, where $f$ is a uniformly predictable process, i.e.

$$
\widetilde{\mathcal{U}(\mathcal{A})}=\left\{\widetilde{L}_{f}: f-\text { a uniformly predictable process }\right\}^{\prime \prime} \text {. }
$$

3. By $\widetilde{\mathfrak{S}(\mathcal{A})}$ we denote the von Neumann algebra on $L^{2}(\mathcal{A})$ generated by all the operators $\widetilde{L}_{f}$, where $f$ is a simple predictable process, i.e.

$$
\widetilde{\mathfrak{S}(\mathcal{A})}=\left\{\widetilde{L}_{f}: f-\text { a simple predictable process }\right\}^{\prime \prime} .
$$

It was shown in [10, Theorem 2.2.6] that the following equalities hold true

$$
\widetilde{\mathcal{E}(\mathcal{A})}=\widetilde{\mathcal{U}(\mathcal{A})}=\widetilde{\mathfrak{S}(\mathcal{A})}=\widetilde{\mathcal{L}(\mathcal{A})}=\widetilde{\mathcal{I}_{l}(\mathcal{A})} .
$$

Now, if $f$ is an arbitrary elementary predictable process of the form

$$
f=\lambda_{0}\left[0_{P}\right]+\sum_{i=1}^{n} \lambda_{i}\left(\sigma_{i}, \tau_{i}\right],
$$

then by linearity

$$
\begin{aligned}
\widetilde{L}_{f} & =\lambda_{0} \mathcal{M}_{0} P+\lambda_{0} L_{\left[0_{P}\right]}+\sum_{i=1}^{n} \lambda_{i} L_{\left(\sigma_{i}, \tau_{i}\right]} \\
& =\lambda_{0} \mathcal{M}_{0} P+\sum_{i=1}^{n} \lambda_{i}\left(\mathcal{M}_{\tau_{i}}-\mathcal{M}_{\sigma_{i}}\right),
\end{aligned}
$$

because $L_{\tau_{i}}=\mathcal{M}_{\tau_{i}}^{\perp}, L_{\left(\sigma_{i}, \tau_{i}\right]}=\mathcal{M}_{\tau_{i}}-\mathcal{M}_{\sigma_{i}}$ and $L_{\left[0_{P}\right]}=0$ by virtue of the assumed right continuity of the filtration. For more details see the proof of Theorem 2.2.5 of [10]. 


\section{RANDOM PARtition of the StOchastic Interval $\left[\tau_{0}, \tau_{\infty}\right]$}

The aim of this section is to introduce the notion of random partition of stochastic interval $\left[\tau_{0}, \tau_{\infty}\right]$ by analogy with the classical case. We shall also characterize the predictable processes associated with these random partitions.

The random time $\tau_{0}$ is the time $\widetilde{0}$ which is the smallest random time while $\tau_{\infty}$ is the time $\widetilde{\infty}$ which is the greatest random time. Recall that the time projection associated with $\tau_{0}$ is the projection $\mathcal{M}_{0}$ and the time projection associated with $\tau_{\infty}$ is the projection 1 .

Definition 4.1. Let $\sigma$ denote a finite sequence of random times:

$$
\tau_{0} \leq \tau_{1} \leq \cdots \leq \tau_{n-1} \leq \tau_{\infty} .
$$

The sequence $\sigma$ is called a random partition of the stochastic interval $\left[\tau_{0}, \tau_{\infty}\right]$. The family of all random partitions of $\left[\tau_{0}, \tau_{\infty}\right]$ is denoted by $\mathcal{P}\left[\tau_{0}, \tau_{\infty}\right]$. Using the identification between the deterministic times and the random times, we obtain that each partition $\theta \in \Theta$ is a random partition, so $\Theta \subset \mathcal{P}\left[\tau_{0}, \tau_{\infty}\right]$.

Let $\sigma=\left\{\tau_{0}, \tau_{1}, \ldots, \tau_{n-1}, \tau_{\infty}\right\}$ be a random partition, we shall call $f$ which has the form

$$
f=\lambda_{0}\left[0_{P}\right]+\sum_{i=1}^{n} \lambda_{i}\left(\tau_{i-1}, \tau_{i}\right],
$$

where $\lambda_{i} \in \mathbb{C}$, for $0 \leq i \leq n$ and $P$ is a projection in $\mathcal{A}_{0}$, the elementary predictable process associated with the random partition $\sigma$. To avoid repeating the phrase " $f$ is an elementary predictable process associated with random partition $\sigma$ " we shall say that $f$ is a $\sigma$-elementary predictable process. Similarly $\sigma$-simple ( $\sigma$-uniformly, $\sigma$-bounded) predictable processes are defined in the entirely analogous way to the simple (resp. uniform, bounded) predictable processes.

Let us start with the following result on $\sigma$-simple predictable processes.

Theorem 4.2. Let $\sigma=\left\{\tau_{0}, \tau_{1}, \ldots, \tau_{n-1}, \tau_{\infty}\right\} \in \mathcal{P}\left[\tau_{0}, \tau_{\infty}\right]$. Then a process $f$ is $\sigma$-simple predictable process if and only if it has the form

$$
f=f_{0} \chi_{\{0\}}+\sum_{i=1}^{n} \alpha_{i}\left(\tau_{i-1}, \tau_{i}\right],
$$

where $\alpha_{i} \in \mathbb{C}$, for $1 \leq i \leq n$ and $f_{0}$ is a finite linear combination of finite products of projections in $\mathcal{A}_{0}$. 
Proof. Suppose that $f$ is a $\sigma$-simple predictable process. Then $f$ is a finite linear combination of finite products of $\sigma$-elementary predictable processes of the form

$$
\lambda_{0}\left[0_{P}\right]+\sum_{i=1}^{n} \lambda_{i}\left(\tau_{i-1}, \tau_{i}\right] .
$$

By the mutual orthogonality of the projections $\left\{\tau_{i-1}(t)-\tau_{i}(t)\right\}_{i=1}^{n}$ for $t \in$ $[0,+\infty]$, we observe that $f_{0}$ is a finite linear combination of finite products of projections in $\mathcal{A}_{0}$ and $f_{t}=\sum_{i=1}^{n} \alpha_{i}\left[\tau_{i-1}(t)-\tau_{i}(t)\right]$ for some $\alpha_{i} \in \mathbb{C}$, $1 \leq i \leq n$. This shows that the process $f$ has the form

$$
f=f_{0} \chi_{\{0\}}+\sum_{i=1}^{n} \alpha_{i}\left(\tau_{i-1}, \tau_{i}\right] .
$$

For the converse, let $P$ and $Q$ be two projections in $\mathcal{A}_{0}$ such that $f_{0}=P Q$. Then there exist $\lambda_{i}, \lambda_{i}^{\prime} \in \mathbb{C}$ such that $\alpha_{i}=\lambda_{i} \lambda_{i}^{\prime}$, for $1 \leq i \leq n$. Put

$$
\begin{aligned}
h & =\left[0_{P}\right]+\sum_{i=1}^{n} \lambda_{i}\left(\tau_{i-1}, \tau_{i}\right], \\
h^{\prime} & =\left[0_{Q}\right]+\sum_{i=1}^{n} \lambda_{i}^{\prime}\left(\tau_{i-1}, \tau_{i}\right] .
\end{aligned}
$$

They are $\sigma$-elementary predictable processes. Once again by the mutual orthogonality of the projections $\left\{\tau_{i-1}(t)-\tau_{i}(t)\right\}_{i=1}^{n}$ for $t \in[0,+\infty]$, we observe that $f=h h^{\prime}$. Using this fact, we see that the result is true for any finite linear combinations of finite products of projections in $\mathcal{A}_{0}$. This means that $f$ is a finite linear combination of finite products of $\sigma$-elementary predictable processes. So $f$ is a $\sigma$-simple predictable process.

Proposition 4.3. Let $\sigma=\left\{\tau_{0}, \tau_{1}, \ldots, \tau_{n-1}, \tau_{\infty}\right\} \in \mathcal{P}\left[\tau_{0}, \tau_{\infty}\right]$ and let $f$ be a $\sigma$-simple predictable process. Then

$$
\widetilde{L}_{f}=\mathcal{M}_{0} f_{0}+\sum_{i=1}^{n} \alpha_{i}\left(\mathcal{M}_{\tau_{i}}-\mathcal{M}_{\tau_{i-1}}\right),
$$

for $\alpha_{i} \in \mathbb{C}, 1 \leq i \leq n$. Furthermore, $\widetilde{L}_{f}$ is a finite linear combination of finite products of operators associated with $\sigma$-elementray predictable processes.

Proof. From Theorem $4.2 f$ has the form

$$
f=f_{0} \chi_{\{0\}}+\sum_{i=1}^{n} \alpha_{i}\left(\tau_{i-1}, \tau_{i}\right],
$$


where $\alpha_{i} \in \mathbb{C}, 1 \leq i \leq n$, and $f_{0}$ is a finite linear combination of finite products of projections in $\mathcal{A}_{0}$. Then

$$
\widetilde{L}_{f}=\mathcal{M}_{0} f_{0}+L_{f_{0} \chi_{\{0\}}}+\sum_{i=1}^{n} \alpha_{i}\left(\mathcal{M}_{\tau_{i}}-\mathcal{M}_{\tau_{i-1}}\right) .
$$

To get the result let us calculate $L_{f_{0} \chi_{\{0\}}}(X)=\int d X_{t} f_{0} \chi_{\{0\}}(t)$, for $X \in$ $L^{2}(\mathcal{A})$. For a partition $\theta$ of $[0,+\infty]$ we have

$S_{\theta}^{l}\left(f_{0} \chi_{\{0\}}, X\right)=\sum_{i=1}^{m}\left(\mathcal{M}_{t_{i}}-\mathcal{M}_{t_{i-1}}\right)(X) f_{0} \chi_{\{0\}}\left(t_{i-1}\right)=\left(\mathcal{M}_{t_{1}}-\mathcal{M}_{0}\right)(X) f_{0}$.

As $\theta$ refines, this expression tends to $\left(\mathcal{M}_{0+}-\mathcal{M}_{0}\right)(X) f_{0}$ which is zero by virtue of the assumed right continuity of the filtration. So

$$
L_{f_{0} \chi_{\{0\}}}(X)=\int d X_{t} f_{0 \chi_{\{0\}}}(t)=0, \quad \text { for each } X \in L^{2}(\mathcal{A}) .
$$

This shows that

$$
\widetilde{L}_{f}=\mathcal{M}_{0} f_{0}+\sum_{i=1}^{n} \alpha_{i}\left(\mathcal{M}_{\tau_{i}}-\mathcal{M}_{\tau_{i-1}}\right) .
$$

Now, let $f_{0}=P Q$ for some projections $P$ and $Q$ in $\mathcal{A}_{0}$. As in the proof of Theorem 4.2, let us consider the following $\sigma$-elementary predictable processes

$$
\begin{aligned}
h & =\left[0_{P}\right]+\sum_{i=1}^{n} \lambda_{i}\left(\tau_{i-1}, \tau_{i}\right], \\
h^{\prime} & =\left[0_{Q}\right]+\sum_{i=1}^{n} \lambda_{i}^{\prime}\left(\tau_{i-1}, \tau_{i}\right],
\end{aligned}
$$

which satisfy $f=h h^{\prime}$. Then

$$
\begin{aligned}
\widetilde{L_{h^{\prime}}} \widetilde{L_{h}}(\cdot) & =\mathcal{M}_{0}\left(\widetilde{L_{h}}(\cdot)\right) Q+\sum_{i=1}^{n} \lambda_{i}^{\prime}\left(\mathcal{M}_{\tau_{i}}-\mathcal{M}_{\tau_{i-1}}\right)\left(\widetilde{L_{h}}(\cdot)\right) \\
& =\mathcal{M}_{0}(\cdot) P Q+\sum_{i=1}^{n} \lambda_{i}^{\prime} \lambda_{i}\left(\mathcal{M}_{\tau_{i}}-\mathcal{M}_{\tau_{i-1}}\right)(\cdot) \\
& =\mathcal{M}_{0}(\cdot) f_{0}+\sum_{i=1}^{n} \alpha_{i}\left(\mathcal{M}_{\tau_{i}}-\mathcal{M}_{\tau_{i-1}}\right)(\cdot)=\widetilde{L}_{f}(\cdot)
\end{aligned}
$$

because of the mutual orthogonality of the projections $\left(\mathcal{M}_{\tau_{i}}-\mathcal{M}_{\tau_{i-1}}\right)_{i=1}^{n}$. Using this fact, we see that the result is true for any finite linear combinations of finite products of projections in $\mathcal{A}_{0}$. This showing that $\widetilde{L}_{f}$ is 
a finite linear combination of finite products of operators associated with $\sigma$-elementary predictable processes.

Corollary 4.4. Let $\sigma=\left\{\tau_{0}, \tau_{1}, \ldots, \tau_{n-1}, \tau_{\infty}\right\} \in \mathcal{P}\left[\tau_{0}, \tau_{\infty}\right]$. Then, a process $f$ is a $\sigma$-simple predictable process if and only if

$$
\widetilde{L}_{f}=\mathcal{M}_{0} f_{0}+\sum_{i=1}^{n} \alpha_{i}\left(\mathcal{M}_{\tau_{i}}-\mathcal{M}_{\tau_{i-1}}\right),
$$

for some $\alpha_{i} \in \mathbb{C}, 1 \leq i \leq n$ and $f_{0}$ is a finite linear combination of finite products of projections in $\mathcal{A}_{0}$.

Proof. Let $f_{0}$ be a finite linear combination of finite products of projections in $\mathcal{A}_{0}$. We define $f$ by

$$
f=f_{0} \chi_{\{0\}}+\sum_{i=1}^{n} \alpha_{i}\left(\tau_{i-1}, \tau_{i}\right],
$$

where $\alpha_{i} \in \mathbb{C}, 1 \leq i \leq n$ and the result follows from Theorem 4.2.

About the $\sigma$-uniformly predictable processes we have the following.

Theorem 4.5. Let $\sigma=\left\{\tau_{0}, \tau_{1}, \ldots, \tau_{n-1}, \tau_{\infty}\right\} \in \mathcal{P}\left[\tau_{0}, \tau_{\infty}\right]$. Then, a process $f$ is $\sigma$-uniformly predictable process if and only if it has the form

$$
f=f_{0} \chi_{\{0\}}+\sum_{i=1}^{n} \alpha_{i}\left(\tau_{i-1}, \tau_{i}\right]
$$

where $\alpha_{i} \in \mathbb{C}, 1 \leq i \leq n$, and $f_{0} \in \mathcal{A}_{0}$.

Proof. Suppose that $f$ is given in above form to show that $f$ is $\sigma$-uniformly predictable processes. In case $f_{0}=P$, where $P$ is a projection in $\mathcal{A}_{0}$, then $f$ is $\sigma$-elementary predictable process and hence is $\sigma$-uniformly predictable process. If $f_{0}$ is a finite linear combination of projections, the process $f$ may be written as a finite linear combination of $\sigma$-elementary predictable process, i.e $f$ is $\sigma$-simple predictable process and hence $\sigma$-uniformly predictable process. Now the operator $f_{0}$ may be written as a linear combination of four positive operators from $\mathcal{A}_{0}$. In turn, each of these can be written as a norm limit of finite linear combinations of its spectral projections. It follows when $f_{0}$ is positive operator that $f$ may be uniformly approximated in the operator norm by a sequence of $\sigma$-simple predictable processes. This means by definition that $f$ is $\sigma$-uniformly predictable process. The result extends to the case of $f_{0}$ not positive by linearity. 
For the converse, we assume that $f$ is $\sigma$-uniformly predictable process. Then $f=\lim _{m \rightarrow+\infty} f^{m}$, where $f^{m}$ are $\sigma$-simple predictable processes, and the limit is uniform. From Theorem 4.2, $f^{m}$ has the form

$$
f^{m}=f_{0}^{m} \chi_{\{0\}}+\sum_{i=1}^{n} \alpha_{i}^{m}\left(\tau_{i-1}, \tau_{i}\right],
$$

where $\left(\alpha_{i}^{m}\right)$ in $\mathbb{C}$ and $\left(f_{0}^{m}\right)$ is a sequence of finite linear combinations of finite products of projections in $\mathcal{A}_{0}$. Thus the sequence $\left(f_{0}^{m}\right)$ converges in operator norm to $f_{0}$ and the sequence $\left\{\sum_{i=1}^{n} \alpha_{i}^{m}\left[\tau_{i-1}(t)-\tau_{i}(t)\right]\right\}$ converges in operator norm to $f_{t}$ for $t \in(0,+\infty]$. This implies that for $i=1,2, \ldots, n$ the sequence $\left\{\alpha_{i}^{m}\left[\tau_{i-1}(t)-\tau_{i}(t)\right]\right\}$ converges in operator norm to $f_{t}\left[\tau_{i-1}(t)-\tau_{i}(t)\right]$ for $t \in(0,+\infty]$ (by virtue of the mutual orthogonality of the projections $\left.\left[\tau_{i-1}(t)-\tau_{i}(t)\right]\right)$. But $\left\{\alpha_{i}^{m}\left[\tau_{i-1}(t)-\tau_{i}(t)\right]\right\}$ is a sequence in the $\mathrm{C}^{*}$-algebra $\mathbb{C}\left[\tau_{i-1}(t)-\tau_{i}(t)\right]$, there exists $\lambda_{i} \in \mathbb{C}$ such that

$$
f_{t}\left[\tau_{i-1}(t)-\tau_{i}(t)\right]=\lambda_{i}\left[\tau_{i-1}(t)-\tau_{i}(t)\right],
$$

and

$$
f_{t}=\sum_{i=1}^{n} f_{t}\left[\tau_{i-1}(t)-\tau_{i}(t)\right]=\sum_{i=1}^{n} \lambda_{i}\left[\tau_{i-1}(t)-\tau_{i}(t)\right] .
$$

Furthermore, by the mutual orthogonality of the projections $\left[\tau_{i-1}(t)-\tau_{i}(t)\right]$ we observe that this decomposition is unique in the sense

$$
f_{t}=\sum_{i=1}^{n} \lambda_{i}\left[\tau_{i-1}(t)-\tau_{i}(t)\right]=\sum_{i=1}^{n} \lambda_{i}^{\prime}\left[\tau_{i-1}(t)-\tau_{i}(t)\right] \Longrightarrow \lambda_{i}=\lambda_{i}^{\prime} .
$$

This shows that the process $f$ has the form

$$
f=f_{0} \chi_{\{0\}}+\sum_{i=1}^{n} \lambda_{i}\left(\tau_{i-1}, \tau_{i}\right]
$$

The result follows.

Note that Theorem 4.5 is still true when replacing the $\sigma$-uniformly predictable process by $\sigma$-bounded predictable process and the proof is essentially the same (using the pointwise convergence in operator norm and the fact that the uniform convergence implies the pointwise). This means that for a fixed random partition $\sigma$, the $\sigma$-uniformly predictable processes and the $\sigma$-bounded predictable processes coincide. So we have the following:

Corollary 4.6. Let $\sigma=\left\{\tau_{0}, \tau_{1}, \ldots, \tau_{n-1}, \tau_{\infty}\right\} \in \mathcal{P}\left[\tau_{0}, \tau_{\infty}\right]$. Then a process $f$ is $\sigma$-uniformly predictable if and only if it is a $\sigma$-bounded predictable process. 
Proposition 4.7. Let $\sigma=\left\{\tau_{0}, \tau_{1}, \ldots, \tau_{n-1}, \tau_{\infty}\right\} \in \mathcal{P}\left[\tau_{0}, \tau_{\infty}\right]$ and let $f$ be $\sigma$-unifomly predictable. Then

$$
\widetilde{L}_{f}=\mathcal{M}_{0} f_{0}+\sum_{i=1}^{n} \alpha_{i}\left(\mathcal{M}_{\tau_{i}}-\mathcal{M}_{\tau_{i-1}}\right) .
$$

Furthermore $\widetilde{L}_{f}$ is a strong limit of a sequence of operators associated with $\sigma$-simple predictable processes.

Proof. From Theorem 4.5, $f$ has the form

$$
f=f_{0} \chi_{\{0\}}+\sum_{i=1}^{n} \alpha_{i}\left(\tau_{i-1}, \tau_{i}\right]
$$

where $\alpha_{i} \in \mathbb{C}, 1 \leq i \leq n$, and $f_{0} \in \mathcal{A}_{0}$. Then

$$
\widetilde{L}_{f}=\mathcal{M}_{0} f_{0}+\sum_{i=1}^{n} \alpha_{i}\left(\mathcal{M}_{\tau_{i}}-\mathcal{M}_{\tau_{i-1}}\right),
$$

since $L_{f_{0} \chi_{\{0\}}}=0$ (by the same manner of the proof of Proposition 4.3). Moving on to the second part we see immediately that $f$ is a uniform limit of a sequence $\left(f_{m}\right)$ of $\sigma$-simple predictable processes which having the form (Theorem 4.2)

$$
f_{m}=f_{0}^{m} \chi_{\{0\}}+\sum_{i=1}^{n} \alpha_{i}^{m}\left(\tau_{i-1}, \tau_{i}\right],
$$

where $\left(\alpha_{i}^{m}\right)$ in $\mathbb{C}$ and $\left(f_{0}^{m}\right)$ is a sequence of finite linear combinations of finite products of projections in $\mathcal{A}_{0}$. From Proposition $4.3, \widetilde{L}_{f_{m}}$ has the form

$$
\widetilde{L}_{f_{m}}=\mathcal{M}_{0} f_{0}^{m}+\sum_{i=1}^{n} \alpha_{i}^{m}\left(\mathcal{M}_{\tau_{i}}-\mathcal{M}_{\tau_{i-1}}\right) .
$$

Note that, since $f$ is a uniform limit of the sequence $\left(f_{m}\right)$ we obtain that the sequence $\left(f_{0}^{m}\right)$ converges in operator norm to $f_{0}$ and for $1 \leq$ $i \leq n$ the sequence $\left\{\alpha_{i}^{m}\left[\tau_{i-1}(t)-\tau_{i}(t)\right]\right\}$ converges in operator norm to $\alpha_{i}\left[\tau_{i-1}(t)-\tau_{i}(t)\right]$, for $t \in(0,+\infty]$. This implies that the sequence $\left(\mathcal{M}_{0}(\cdot) f_{0}^{m}\right)$ converges strongly to $\left(\mathcal{M}_{0}(\cdot) f_{0}\right)$ on $L^{2}(\mathcal{A})$ and the sequence $\left(\alpha_{i}^{m}\right)$ converges to $\alpha_{i}$, for $1 \leq i \leq n$. This shows that

$$
\sum_{i=1}^{n} \alpha_{i}^{m}\left(\mathcal{M}_{\tau_{i}}-\mathcal{M}_{\tau_{i-1}}\right) \longrightarrow m \sum_{i=1}^{n} \alpha_{i}\left(\mathcal{M}_{\tau_{i}}-\mathcal{M}_{\tau_{i-1}}\right),
$$

in the strong operator to topology on $B\left(L^{2}(\mathcal{A})\right)$ and hence

$$
\mathcal{M}_{0} f_{0}^{m}+\sum_{i=1}^{n} \alpha_{i}^{m}\left(\mathcal{M}_{\tau_{i}}-\mathcal{M}_{\tau_{i-1}}\right) \longrightarrow_{m} \mathcal{M}_{0} f_{0}+\sum_{i=1}^{n} \alpha_{i}\left(\mathcal{M}_{\tau_{i}}-\mathcal{M}_{\tau_{i-1}}\right),
$$


in the strong operator topology, i.e $\widetilde{L}_{f_{m}} \longrightarrow_{m} \widetilde{L}_{f}$ strongly, as required.

Corollary 4.8. Let $\sigma=\left\{\tau_{0}, \tau_{1}, \ldots, \tau_{n-1}, \tau_{\infty}\right\} \in \mathcal{P}\left[\tau_{0}, \tau_{\infty}\right]$ and $f$ is a $\sigma$ unifomly predictable process. Then $\widetilde{L}_{f}$ is a strong limit of a sequence of finite linear combinations of finite products of operators associated with $\sigma$ elementary predictable processes.

This follows immediately from Proposition 4.7 and Proposition 4.3.

\section{RANDOM PARTITIONS AND RELATED ALGEBRAS}

In this section we shall use random partitions to examine mutual relations between operator algebras connected with stochastic integrals of predictable processes. Also we shall identify the algebras connected with the stochastic integrals of $\sigma$-predictable processes. Let us start with the following definition.

Definition 5.1. Let $\sigma=\left\{\tau_{0}, \tau_{1}, \ldots, \tau_{n-1}, \tau_{\infty}\right\}$ be a fixed random partition.

1. By $\mathcal{E}^{\sigma}$ we denote the von Neumann algebra on $L^{2}(\mathcal{A})$ generated by all the operators $\widetilde{L}_{f}$ where $f$ is a $\sigma$-elementary predictable process, i.e.

$$
\mathcal{E}^{\sigma}=\left\{\widetilde{L}_{f}: f \text { is a } \sigma \text {-elementary predictable process }\right\}^{\prime \prime} .
$$

2. By $\mathfrak{S}^{\sigma}$ we denote the von Neumann algebra on $L^{2}(\mathcal{A})$ generated by all the operators $\widetilde{L}_{f}$, where $f$ is a $\sigma$-simple predictable process, i.e.

$$
\mathfrak{S}^{\sigma}=\left\{\widetilde{L}_{f}: f \text { is a } \sigma \text {-simple predictable process }\right\}^{\prime \prime} .
$$

3. By $\mathcal{U}^{\sigma}$ we denote the von Neumann algebra on $L^{2}(\mathcal{A})$ generated by all the operators $\widetilde{L}_{f}$, where $f$ is a $\sigma$-uniformly predictable process, i.e.

$$
\mathcal{U}^{\sigma}=\left\{\widetilde{L}_{f}: f \text { is a } \sigma \text {-uniformly predictable process }\right\}^{\prime \prime} .
$$

As an immediate consequences we have the following.

Corollary 5.2. For a fixed random partition $\sigma=\left\{\tau_{0}, \tau_{1}, \ldots, \tau_{n-1}, \tau_{\infty}\right\}$, the following equalities hold true

$$
\mathcal{E}^{\sigma}=\mathfrak{S}^{\sigma}=\mathcal{U}^{\sigma}
$$

Proof. By Definition 5.1 we have

$$
\mathcal{E}^{\sigma} \subseteq \mathfrak{S}^{\sigma} \subseteq \mathcal{U}^{\sigma}
$$

From Proposition 4.3 we have $\mathfrak{S}^{\sigma} \subseteq \mathcal{E}^{\sigma}$ and from Proposition 4.7 and Corollary 4.8 we have $\mathcal{U}^{\sigma} \subseteq \mathfrak{S}^{\sigma} \subseteq \mathcal{E}^{\sigma}$. So $\mathcal{E}^{\sigma}=\mathfrak{S}^{\sigma}=\mathcal{U}^{\sigma}$. 
Corollary 5.3. The following equalities hold true

$$
\left(\bigcup_{\sigma} \mathcal{E}^{\sigma}\right)^{\prime \prime}=\left(\bigcup_{\sigma} \mathfrak{S}^{\sigma}\right)^{\prime \prime}=\left(\bigcup_{\sigma} \mathcal{U}^{\sigma}\right)^{\prime \prime}
$$

The following lemma identifies the von Neumann algebra $\mathcal{E}^{\sigma}$ associated with the random partition $\sigma$.

Lemma 5.4. For each $\sigma=\left\{\tau_{0}, \tau_{1}, \ldots, \tau_{n-1}, \tau_{\infty}\right\} \in \mathcal{P}\left[\tau_{0}, \tau_{\infty}\right]$, we have

$$
\mathcal{E}^{\sigma}=\mathcal{M}_{0} \mathcal{A}_{0} \oplus \bigoplus_{i=1}^{n} \mathbb{C}\left(\mathcal{M}_{\tau_{i}}-\mathcal{M}_{\tau_{i-1}}\right)
$$

Proof. For a fixed random partition $\sigma=\left\{\tau_{0}, \tau_{1}, \ldots, \tau_{n-1}, \tau_{\infty}\right\}$ and a fixed $\sigma$-elementary predictable process $f$ with the form

$$
f=\lambda_{0}\left[0_{P}\right]+\sum_{i=1}^{n} \lambda_{i}\left(\tau_{i-1}, \tau_{i}\right]
$$

where $P$ is a projection in $\mathcal{A}_{0}$ and $\lambda_{i} \in \mathbb{C}$, for $0 \leq i \leq n$. We have

$$
\widetilde{L}_{f}=\lambda_{0} \mathcal{M}_{0} P+\sum_{i=1}^{n} \lambda_{i}\left(\mathcal{M}_{\tau_{i}}-\mathcal{M}_{\tau_{i-1}}\right),
$$

which belongs to

$$
\mathcal{M}_{0} \mathcal{A}_{0} \oplus \bigoplus_{i=1}^{n} \mathbb{C}\left(\mathcal{M}_{\tau_{i}}-\mathcal{M}_{\tau_{i-1}}\right) .
$$

By mutual orthogonality of the projections $\left(\mathcal{M}_{\tau_{i}}-\mathcal{M}_{\tau_{i-1}}\right)_{i=1}^{n}$ the same can be said of any element of the $*$-algebra generated by the $\widetilde{L}_{f}$. Thus

$$
\mathcal{E}^{\sigma} \subseteq \mathcal{M}_{0} \mathcal{A}_{0} \oplus \bigoplus_{i=1}^{n} \mathbb{C}\left(\mathcal{M}_{\tau_{i}}-\mathcal{M}_{\tau_{i-1}}\right)
$$

For the next part we note that each element $y$ of the algebra

$$
\mathcal{M}_{0} \mathcal{A}_{0} \oplus \bigoplus_{i=1}^{n} \mathbb{C}\left(\mathcal{M}_{\tau_{i}}-\mathcal{M}_{\tau_{i-1}}\right)
$$

which has the form

$$
\alpha_{0} \mathcal{M}_{0} P+\sum_{i=1}^{n} \alpha_{i}\left(\mathcal{M}_{\tau_{i}}-\mathcal{M}_{\tau_{i-1}}\right)
$$

where $\alpha_{i} \in \mathbb{C}$, for $0 \leq i \leq n$, and $P$ is a projection in $\mathcal{A}_{0}$, is an operator $\widetilde{L}_{f}$ in $\mathcal{E}^{\sigma}$ for some $\sigma$-elementary predictable process $f$. If $P$ is a finite linear combination of projections in $\mathcal{A}_{0}$, then $y$ may be written as a finite 
linear combination of operators associated with $\sigma$-elementary predictable processes and hence $y \in \mathcal{E}^{\sigma}$. Now the operator $P$ may be written as a linear combination of four positive operators from $\mathcal{A}_{0}$. In turn, each of these can be written as a norm limit of finite linear combinations of its spectral projections. It follows that when $P$ is a positive operator, $y$ may be approximated in the strong operator topology by a sequence of linear combinations of operators associated with $\sigma$-elementary predictable processes, so $y \in \mathcal{E}^{\sigma}$. The result extends to the case of $P$ not positive by linearity. This shows that

$$
\mathcal{E}^{\sigma}=\mathcal{M}_{0} \mathcal{A}_{0} \oplus \bigoplus_{i=1}^{n} \mathbb{C}\left(\mathcal{M}_{\tau_{i}}-\mathcal{M}_{\tau_{i-1}}\right)
$$

Remark 5.5. The second part of the above proof shows that each element of $\mathcal{E}^{\sigma}$ is a strong operator limit of a sequence of linear combinations of operators associated with $\sigma$-elementary predictable processes. This means that each element of $\mathcal{E}^{\sigma}$ is a strong operator limit of a sequence of operators associated with $\sigma$-simple predictable processes.

Theorem 5.6. The following equalities hold true

1. $\widetilde{\mathcal{E}(\mathcal{A})}=\left(\bigcup_{\sigma} \mathcal{E}^{\sigma}\right)^{\prime \prime}$.

2. $\widetilde{\mathfrak{S}(\mathcal{A})}=\left(\bigcup_{\sigma} \mathfrak{S}^{\sigma}\right)^{\prime \prime}$.

3. $\widetilde{\mathcal{U}(\mathcal{A})}=\left(\bigcup_{\sigma} \mathcal{U}^{\sigma}\right)^{\prime \prime}$.

Proof. 1. It is clear that $\left\{\mathcal{E}^{\sigma}: \sigma \in \mathcal{P}\left[\tau_{0}, \tau_{\infty}\right]\right\}$ is a family of von Neumann subalgebras of $\widetilde{\mathcal{E}(\mathcal{A})}$. So $\left(\bigcup_{\sigma} \mathcal{E}^{\sigma}\right)^{\prime \prime} \subseteq \widehat{\mathcal{E}(\mathcal{A})}$. Let $f$ be an elementary predictable process, then there exist a random partition $\sigma \in \mathcal{P}\left[\tau_{0}, \tau_{\infty}\right]$ such that $f$ is $\sigma$-elementary predictable process and so $\widetilde{L}_{f} \in \mathcal{E}^{\sigma}$. This shows that $\widetilde{L}_{f} \in\left(\bigcup_{\sigma} \mathcal{E}^{\sigma}\right)^{\prime \prime}$ and hence $\widetilde{\mathcal{E}(\mathcal{A})} \subseteq\left(\bigcup_{\sigma} \mathcal{E}^{\sigma}\right)^{\prime \prime}$, which proves the equality $\widetilde{\mathcal{E}(\mathcal{A})}=\left(\bigcup_{\sigma} \mathcal{E}^{\sigma}\right)^{\prime \prime}$

2. Let $f$ be a simple predictable process. Then by definition $f$ has the form $f=\sum_{i=1}^{r} f_{1}^{(i)} \cdots f_{k_{i}}^{(i)}$, where $f_{1}^{(i)}, \ldots, f_{k_{i}}^{(i)}$ are elementary predictable processes. For each $1 \leq i \leq r$ and $1 \leq k \leq k_{i}$, there exist a random partition $\sigma_{k}^{i} \in \mathcal{P}\left[\tau_{0}, \tau_{\infty}\right]$ such that $f_{k}^{(i)}$ is $\sigma_{k}^{i}$-elementary predictable process. By Theorem 3.2(1), we have that

$$
L_{f}=\sum_{i=1}^{r} L_{f_{k_{i}}^{(i)}} \ldots L_{f_{1}^{(i)}} .
$$


Now, we define new $\sigma_{k}^{i}$-elementary predictable processes, say $g_{k}^{(i)}$ for $1 \leq$ $i \leq r$ and $1 \leq k \leq k_{i}$, which differ from $f_{k}^{(i)}$ only at point 0 , namely so that $g_{k}^{(i)}(0)=f_{k_{i}-k+1}^{(i)}(0)$. Then $L_{g_{k}^{(i)}}=L_{f_{k}^{(i)}}$ and

$$
\widetilde{L}_{g_{k}^{(i)}}=\mathcal{M}_{0} g_{k}^{(i)}(0)+L_{g_{k}^{(i)}}=\mathcal{M}_{0} f_{k_{i}-k+1}^{(i)}(0)+L_{f_{k}^{(i)}} .
$$

Furthermore,

$$
\begin{aligned}
\sum_{i=1}^{r} \widetilde{L}_{g_{k_{i}}^{(i)}} \cdots \widetilde{L}_{g_{1}^{(i)}} & =\sum_{i=1}^{r}\left[g_{k_{i}}^{(i)}(0) \mathcal{M}_{0}+L_{g_{k_{i}}^{(i)}}\right] \cdots\left[g_{1}^{(i)}(0) \mathcal{M}_{0}+L_{g_{1}^{(i)}}\right] \\
& =\sum_{i=1}^{r}\left[g_{k_{i}}^{(i)}(0) \mathcal{M}_{0}+L_{f_{k_{i}}^{(i)}}\right] \cdots\left[g_{1}^{(i)}(0) \mathcal{M}_{0}+L_{f_{1}^{(i)}}\right] \\
& =\sum_{i=1}^{r} g_{k_{i}}^{(i)}(0) \cdots g_{1}^{(i)}(0) \mathcal{M}_{0}+\sum_{i=1}^{r} L_{f_{k_{i}}^{(i)}} \cdots L_{f_{1}^{(i)}} \\
& =\sum_{i=1}^{r} f_{1}^{(i)}(0) \cdots f_{k_{i}}^{(i)}(0) \mathcal{M}_{0}+\sum_{i=1}^{r} L_{f_{k_{i}}^{(i)}} \cdots L_{f_{1}^{(i)}} \\
& =f_{0} \mathcal{M}_{0}+L_{f}=\mathcal{M}_{0} f_{0}+L_{f}=\widetilde{L}_{f},
\end{aligned}
$$

because any terms involving products of $\mathcal{M}_{0}$ with $L_{f_{k}^{(i)}}$ will vanish (for more details see the proof of $\left[8\right.$, Theorem 5.5]). Since clearly $\widetilde{L}_{g_{k}^{(i)}} \in\left(\bigcup_{\sigma} \mathcal{E}^{\sigma}\right)^{\prime \prime}=$ $\left(\bigcup_{\sigma} \mathfrak{S}^{\sigma}\right)^{\prime \prime}$ for $1 \leq i \leq r$ and $1 \leq k \leq k_{i}$, we obtain that $\widetilde{L}_{f} \in\left(\bigcup_{\sigma} \mathfrak{S}^{\sigma}\right)^{\prime \prime}$ and hence $\widetilde{\mathfrak{S}(\mathcal{A})} \subseteq\left(\bigcup_{\sigma} \mathfrak{S}^{\sigma}\right)^{\prime \prime}$. But $\left(\bigcup_{\sigma} \mathfrak{S}^{\sigma}\right)^{\prime \prime} \subseteq \widehat{\mathfrak{S}(\mathcal{A})}$. We get the equality $\widetilde{\mathfrak{S}(\mathcal{A})}=\left(\bigcup_{\sigma} \mathfrak{S}^{\sigma}\right)^{\prime \prime}$

3. Let $f$ be a uniformly predictable process. Then there exists a sequence $\left(f_{n}\right)$ of simple predictable processes converges to $f$ uniformly. Thus $L_{f}=$ $\lim _{n \rightarrow \infty} L_{f_{n}}$ strongly (see [10, Proposition 2.1.3]). Note that $\mathcal{M}_{0}(\cdot) f_{n}(0)$ converges strongly to $\mathcal{M}_{0}(\cdot) f_{0}$ (since we have the sequence $f_{n}(0)$ converges to $f_{0}$ in operator norm). Combining with above we get $\widetilde{L}_{f}=\lim _{n \rightarrow \infty} \widetilde{L}_{f_{n}}$ strongly. We know from the part 2 that $\widetilde{L}_{f_{n}} \in\left(\bigcup_{\sigma} \mathfrak{S}^{\sigma}\right)^{\prime \prime}$ for each $n$ and from Corollary 5.3 that $\left(\bigcup_{\sigma} \mathfrak{S}^{\sigma}\right)^{\prime \prime}=\left(\bigcup_{\sigma} \mathcal{U}^{\sigma}\right)^{\prime \prime}$, so $\widetilde{L}_{f_{n}} \in\left(\bigcup_{\sigma} \mathcal{U}^{\sigma}\right)^{\prime \prime}$ for each $n$ and hence $\widetilde{L}_{f} \in\left(\bigcup_{\sigma} \mathcal{U}^{\sigma}\right)^{\prime \prime}$, which proves the inclusion $\widetilde{\mathcal{U}(\mathcal{A})} \subseteq\left(\bigcup_{\sigma} \mathcal{U}^{\sigma}\right)^{\prime \prime}$. But we know that $\left(\bigcup_{\sigma} \mathcal{U}^{\sigma}\right)^{\prime \prime} \subseteq \widetilde{\mathcal{U}(\mathcal{A})}$, as claimed.

Corollary 5.7. The following equalities hold true

$$
\widetilde{\mathcal{E}(\mathcal{A})}=\widetilde{\mathfrak{S}(\mathcal{A})}=\widetilde{\mathcal{U}(\mathcal{A})} \text {. }
$$


This follows immediately from Theorem 5.6 and Corollary 5.3.

Proposition 5.8. The following equalities hold true

$$
\widetilde{\mathcal{E}(\mathcal{A})}=\widetilde{\mathfrak{S}(\mathcal{A})}=\widetilde{\mathcal{U}(\mathcal{A})}=\left\{\mathcal{M}_{0} \mathcal{A}_{0}, \mathcal{M}_{\tau}: \tau \text { is a random time }\right\}^{\prime \prime} .
$$

Proof. For each random time $\tau$, the stochastic interval $f=\left(\tau, \tau_{\infty}\right]$ is an elementary predictable process with $\widetilde{L}_{f}=\mathcal{M}_{\tau_{\infty}}-\mathcal{M}_{\tau}=\mathcal{M}_{\tau}^{\perp}$. Thus $\mathcal{M}_{\tau}^{\perp} \in$ $\widetilde{\mathcal{E}(\mathcal{A})}$ and hence $\mathcal{M}_{\tau} \in \widetilde{\mathcal{E}(\mathcal{A})}$ for each random time $\tau$. From Lemma 5.4, we have

$$
\mathcal{E}^{\sigma}=\mathcal{M}_{0} \mathcal{A}_{0} \oplus \bigoplus_{i=1}^{n} \mathbb{C}\left(\mathcal{M}_{\tau_{i}}-\mathcal{M}_{\tau_{i-1}}\right)
$$

for each random partition $\sigma=\left\{\tau_{0}, \tau_{1}, \ldots, \tau_{n-1}, \tau_{\infty}\right\}$. The inclusion $\mathcal{E}^{\sigma} \subset$ $\widetilde{\mathcal{E}(\mathcal{A})}$ entails $\mathcal{M}_{0} \mathcal{A}_{0} \subset \widetilde{\mathcal{E}(\mathcal{A})}$. Thus

$$
\left\{\mathcal{M}_{0} \mathcal{A}_{0}, \mathcal{M}_{\tau}: \tau \text { is a random time }\right\}^{\prime \prime} \subset \widetilde{\mathcal{E}(\mathcal{A})} .
$$

But for each random partition $\sigma$, we have

$$
\mathcal{E}^{\sigma} \subseteq\left\{\mathcal{M}_{0} \mathcal{A}_{0}, \mathcal{M}_{\tau}: \tau \text { is a random time }\right\}^{\prime \prime} .
$$

By using Theorem 5.6(1) we get that

$$
\widetilde{\mathcal{E}(\mathcal{A})} \subseteq\left\{\mathcal{M}_{0} \mathcal{A}_{0}, \mathcal{M}_{\tau}: \tau \text { is a random time }\right\}^{\prime \prime},
$$

and hence

$$
\widetilde{\mathcal{E}(\mathcal{A})}=\left\{\mathcal{M}_{0} \mathcal{A}_{0}, \mathcal{M}_{\tau}: \tau \text { is a random time }\right\}^{\prime \prime}
$$

as claimed.

We conclude this section by the following result concerning the sequences in the algebra $\mathcal{E}^{\sigma}$. For a fixed random partition $\sigma=\left\{\tau_{0}, \tau_{1}, \ldots, \tau_{n-1}, \tau_{\infty}\right\}$. The sequence $\left(b_{k}\right)$ in $\mathcal{E}^{\sigma}$ is a combination of two sequences in $\mathcal{B}\left(L^{2}(\mathcal{A})\right)$, i.e.

$$
b_{k}=\mathcal{M}_{0} a_{k}+\sum_{i=1}^{n} \lambda_{i}^{(k)}\left(\mathcal{M}_{\tau_{i}}-\mathcal{M}_{\tau_{i-1}}\right),
$$

where $\left(a_{k}\right)$ is a sequence in $\mathcal{A}_{0}$ and $\left(\lambda_{i}^{(k)}\right)$ is a sequence in $\mathbb{C}$, for $0 \leq i \leq n$. The next result gives us an idea of the conditions that make the sequence in $\mathcal{E}^{\sigma}$ is Cauchy in the strong operator topology.

Proposition 5.9. The sequence $\left(b_{k}\right)$ is strongly Cauchy and $\left(a_{k}\right)$ is a uniformly bounded sequence if and only if the sequences $\left(a_{k}\right)$ and $\left(\lambda_{i}^{(k)}\left(\mathcal{M}_{\tau_{i}}-\mathcal{M}_{\tau_{i-1}}\right)\right), i=1,2, \ldots, n$ are strongly Cauchy. 
Proof. We have $\left(b_{k}\right)$ is strongly Cauchy. Then the sequence

$$
b_{k}(\mathbf{1})=\mathcal{M}_{0}(\mathbf{1}) a_{k}+\sum_{i=1}^{n} \lambda_{i}^{(k)}\left(\mathcal{M}_{\tau_{i}}-\mathcal{M}_{\tau_{i-1}}\right)(\mathbf{1})=a_{k},
$$

is Cauchy in $L^{2}(\mathcal{A})$. But the sequence $a_{k}$ is uniformly bounded, it follows from [7, Lemma 1] that $a_{k}$ is strongly Cauchy in $\mathcal{A}$ and hence $\mathcal{M}_{0}(\cdot) a_{k}$ is strongly Cauchy in $\mathcal{B}\left(L^{2}(\mathcal{A})\right)$. This implies that $\sum_{i=1}^{n} \lambda_{i}^{(k)}\left(\mathcal{M}_{\tau_{i}}-\mathcal{M}_{\tau_{i-1}}\right)$ is strongly Cauchy. By mutual orthogonality of the projections $\left(\mathcal{M}_{\tau_{i}}-\mathcal{M}_{\tau_{i-1}}\right)_{i=1}^{n}$ and the continuity of the multiplication in the strong operator topology, we obtain that each sequence

$$
\left(\lambda_{i}^{(k)}\left(\mathcal{M}_{\tau_{i}}-\mathcal{M}_{\tau_{i-1}}\right)\right),
$$

is strongly Cauchy for $i=1,2, \ldots, n$. For the converse, we have $\left(\lambda_{i}^{(k)}\left(\mathcal{M}_{\tau_{i}}-\mathcal{M}_{\tau_{i-1}}\right)\right)$ for $i=1,2, \ldots, n$ and $\left(a_{k}\right)$ are strongly Cauchy. Then $\left(a_{k}\right)$ is uniformly bounded and the sequences $\left(\mathcal{M}_{0} a_{k}\right)$ is strongly Cauchy. This gives that the sequence

$$
\mathcal{M}_{0} a_{k}+\sum_{i=1}^{n} \lambda_{i}^{(k)}\left(\mathcal{M}_{\tau_{i}}-\mathcal{M}_{\tau_{i-1}}\right)
$$

is strongly Cauchy.

In other words, according to Theorem 4.5 the above proposition can be formulated in the following form:

Corollary 5.10. Let $\sigma=\left\{\tau_{0}, \tau_{1}, \ldots, \tau_{n-1}, \tau_{\infty}\right\} \in \mathcal{P}\left[\tau_{0}, \tau_{\infty}\right]$ and let $\left(f_{k}\right)$ be a sequence of $\sigma$-uniformly predictable processes. Then the sequence $\left(\widetilde{L}_{f_{k}}\right)$ is strongly Cauchy and $\left(f_{0}^{k}\right)$ is uniformly bounded if and only if the sequences $\left(f_{0}^{k}\right)$ and $\left(\widetilde{L}_{f_{k}}-\mathcal{M}_{0} f_{0}^{k}\right)$ are strongly Cauchy.

\section{RANDOM PARTITIONS AND SIMPLIFICATION}

In this section, we look at the correspondence between simple $\mathcal{A}$-valued adapted processes and $\sigma$-uniformly predictable processes. Also we show that the von Neumann algebra $\mathcal{U}^{\sigma}$ is an image of $*$-preserving strongly continuous positive linear map defined on the product von Neumann algebra of the filtration $\left(\mathcal{A}_{t}\right), t \in[0,+\infty]$.

Let us define the von Neumann algebra

$$
\widetilde{\mathcal{A}}=\prod_{t \in[0,+\infty]} \mathcal{A}_{t}
$$


the product von Neumann algebra for the algebras $\left(\mathcal{A}_{t}\right), t \in[0,+\infty]$ (see $[9$, Part1, Chapter 2, Section 2]). We can consequently view $\mathcal{A}$-valued adapted processes as elements of $\widetilde{\mathcal{A}}$, and a random time is a projection in $\widetilde{\mathcal{A}}$.

We recall some facts about the simplification of $\mathcal{A}$-valued processes. Firstly, note that a simple $\mathcal{A}$-valued process $f$ has the form

$$
f=\sum_{i=1}^{n} z_{t_{i-1}} \chi_{\left[t_{i-1}, t_{i}\right)}+z_{t_{n}} \chi_{\{+\infty\}},
$$

where $z_{t_{0}}, \ldots, z_{t_{n}} \in \mathcal{A}$ and $0=t_{0}<t_{1}<\cdots<t_{n}=+\infty$. $f$ is adapted if $z_{t_{i}} \in \mathcal{A}_{t_{i}}$

Definition 6.1. For a partition $\theta=\left\{0=t_{0}<t_{1}<\ldots<t_{n}=+\infty\right\}$ and an arbitrary $\mathcal{A}$-valued process $f$ let us define the "simplification" $f_{\theta}$ of $f$ by

$$
f_{\theta}=\sum_{i=1}^{n} f_{t_{i-1}} \chi_{\left[t_{i-1}, t_{i}\right)}+f_{+\infty} \chi_{\{+\infty\}}
$$

The class of all adapted $\mathcal{A}$-valued processes simplified by $\theta$ is denoted by $\widetilde{\mathcal{A}_{\theta}}$, i.e.

$$
\widetilde{\mathcal{A}_{\theta}}=\left\{f_{\theta}: f \in \widetilde{\mathcal{A}}\right\} .
$$

Clear that $\widetilde{\mathcal{A}_{\theta}}$ is a $*$-subalgebra of $\widetilde{\mathcal{A}}$. Furthermore, $\widetilde{\mathcal{A}_{\theta}}$ is a von Neumann algebra for each $\theta \in \Theta$ and there exists an ultraweakly continuous *-homomorphism $\theta: \widetilde{\mathcal{A}} \longrightarrow \widetilde{\mathcal{A}_{\theta}}$ defined by

$$
\theta(f)=f_{\theta} .
$$

For more details and the proof see Corollary 3.8 of [6].

The above definition leads to a correspondence between the elements of $\widetilde{\mathcal{A}_{\theta}}$ and the $\sigma$-uniformly predictable processes $(\theta$ and $\sigma$ having the same number of elements). Indeed, if $f \in \widetilde{\mathcal{A}_{\theta}}$, then $f$ has the form

$$
f=\sum_{i=1}^{n} f_{t_{i-1}} \chi_{\left[t_{i-1}, t_{i}\right)}+f_{t_{n}} \chi_{\{+\infty\}} .
$$

The random times $\tau_{0}=\widetilde{t_{0}}<\widetilde{t_{1}}<\ldots<\widetilde{t_{n}}=\tau_{\infty}$ form a random partition of $\left[\tau_{0}, \tau_{\infty}\right]$ and according to Theorem 4.5 we can define a uniformly predictable process $h$ associated with this random partition in the form

$$
h=f_{t_{0}} \chi_{\left\{t_{0}\right\}}+\sum_{i=1}^{n} \varphi\left(f_{t_{i}}\right)\left(\widetilde{t_{i-1}}, \widetilde{t_{i}}\right] .
$$


On the other hand, let $f$ be a $\sigma$-uniformly predictable process. Then $f$ has the form

$$
f=f_{t_{0}} \chi_{\left\{t_{0}\right\}}+\sum_{i=1}^{n} \alpha_{i}\left(\tau_{i-1}, \tau_{i}\right],
$$

for $\alpha_{i} \in \mathbb{C}, 1 \leq i \leq n$, and $f_{t_{0}} \in \mathcal{A}_{0}$. Let $\theta=\left\{0=t_{0}<t_{1}<\cdots<t_{n}=\right.$ $+\infty\} \in \Theta$, we define an $\mathcal{A}$-valued adapted process $h$ by

$$
h=\sum_{i=1}^{n} f_{t_{i-1}}\left[t_{i-1}, t_{i}\right)+f_{t_{n}} \chi_{\{+\infty\}}=\theta(f),
$$

which is an element of $\widetilde{\mathcal{A}_{\theta}}$.

Definition 6.2. For each random partition $\sigma=\left\{\tau_{0}, \tau_{1}, \ldots, \tau_{n-1}, \tau_{\infty}\right\}$ and $\theta=\left\{t_{0}, t_{1}, \ldots, t_{n}\right\} \in \Theta$ we define the map $\mathcal{S}_{\theta}^{\sigma}: \widetilde{\mathcal{A}} \longrightarrow \mathcal{B}\left(L^{2}(\mathcal{A})\right)$ by:

$$
\mathcal{S}_{\theta}^{\sigma}(f)=\mathcal{M}_{0} f_{t_{0}}+\sum_{i=1}^{n} \varphi\left(f_{t_{i}}\right)\left(\mathcal{M}_{\tau_{i}}-\mathcal{M}_{\tau_{i-1}}\right) \text {. }
$$

Remark 6.3. Taking into account the results of the previous section, we see the following:

1. For each $f \in \widetilde{\mathcal{A}}$ we have $\mathcal{S}_{\theta}^{\sigma}(f) \in \mathcal{E}^{\sigma}$. Furthermore, $\mathcal{S}_{\theta}^{\sigma}(f)$ is an integral of a $\sigma$-uniformly predictable process $g$ given by

$$
g=f_{t_{0}} \chi_{\left\{t_{0}\right\}}+\sum_{i=1}^{n} \varphi\left(f_{t_{i}}\right)\left(\tau_{i-1}, \tau_{i}\right] .
$$

2. $\mathcal{S}_{\theta}^{\sigma}=\left.\mathcal{S}_{\theta}^{\sigma}\right|_{\widetilde{\mathcal{A}_{\theta}}} \cdot \theta$, where $\left.\mathcal{S}_{\theta}^{\sigma}\right|_{\widetilde{\mathcal{A}_{\theta}}}$ is the restriction of $\mathcal{S}_{\theta}^{\sigma}$ on $\widetilde{\mathcal{A}_{\theta}}$.

Lemma 6.4. The map $S_{\theta}^{\sigma}: \widetilde{\mathcal{A}} \longrightarrow \mathcal{B}\left(L^{2}(\mathcal{A})\right)$ is:

1. *-preserving positive linear onto $\mathcal{E}^{\sigma}$;

2. strong operator continuous.

Proof. 1. The linearity is obvious. Let $f=\left(f_{t}\right) \in \widetilde{\mathcal{A}}$. Then

$$
\begin{aligned}
{\left[S_{\theta}^{\sigma}(f)\right]^{*} } & =\left[\mathcal{M}_{0} f_{t_{0}}+\sum_{i=1}^{n} \varphi\left(f_{t_{i}}\right)\left(\mathcal{M}_{\tau_{i}}-\mathcal{M}_{\tau_{i-1}}\right)\right]^{*} \\
& =\left(\mathcal{M}_{0} f_{t_{0}}\right)^{*}+\sum_{i=1}^{n} \overline{\varphi\left(f_{t_{i}}\right)}\left(\mathcal{M}_{\tau_{i}}-\mathcal{M}_{\tau_{i-1}}\right) \\
& =\mathcal{M}_{0} f_{t_{0}}^{*}+\sum_{i=1}^{n} \varphi\left(f_{t_{i}}^{*}\right)\left(\mathcal{M}_{\tau_{i}}-\mathcal{M}_{\tau_{i-1}}\right)=\mathcal{S}_{\theta}^{\sigma}\left(f^{*}\right),
\end{aligned}
$$


because $\varphi$ is a positive linear functional and $\left(\mathcal{M}_{t} a_{t}\right)^{*}=\mathcal{M}_{t} a_{t}^{*}$ for $t \in$ $[0,+\infty]$. To show that $\mathcal{S}_{\theta}^{\sigma}$ is a positive, let $f=\left(f_{t}\right) \in(\widetilde{\mathcal{A}})^{+}$. Then $f_{t} \in \mathcal{A}_{t}^{+}$ for each $t \in[0,+\infty]$ and hence $\varphi\left(f_{t}\right) \geq 0$ for each $t \in[0,+\infty]$, also we obtain that $\mathcal{M}_{0} f_{0} \geq 0$. Consequently, we get that $S_{\theta}^{\sigma}(f) \geq 0$.

To prove that the map $S_{\theta}^{\sigma}$ is onto $\mathcal{E}^{\sigma}$, let $y \in \mathcal{E}^{\sigma}$ then according to Lemma 5.4 we have

$$
y=\mathcal{M}_{0} b_{0}+\sum_{i=1}^{n} \lambda_{i}\left(\mathcal{M}_{\tau_{i}}-\mathcal{M}_{\tau_{i-1}}\right),
$$

for some $b_{0} \in \mathcal{A}_{0}$ and $\lambda_{i} \in \mathbb{C}$, for $i=1,2, \ldots, n$. Put $f=\left(f_{t}\right)$ such that $f_{t_{0}}=b_{0}, f_{t_{1}}=\lambda_{1} \mathbf{1}, f_{t_{2}}=\lambda_{2} \mathbf{1}, \ldots, f_{t_{n}}=\lambda_{n} \mathbf{1}$ and the rest is zero. Then $f=\left(f_{t}\right) \in \widetilde{\mathcal{A}}$ and

$$
\begin{aligned}
S_{\theta}^{\sigma}(f) & =\mathcal{M}_{0} f_{t_{0}}+\sum_{i=1}^{n} \varphi\left(\lambda_{i} \mathbf{1}\right)\left(\mathcal{M}_{\tau_{i}}-\mathcal{M}_{\tau_{i-1}}\right), \\
& =\mathcal{M}_{0} b_{0}+\sum_{i=1}^{n} \lambda_{i}\left(\mathcal{M}_{\tau_{i}}-\mathcal{M}_{\tau_{i-1}}\right)=y .
\end{aligned}
$$

2. Suppose that $f_{i}=\left(f_{t}^{(i)}\right) \longrightarrow g=\left(g_{t}\right)$ in the strong operator topology in $\widetilde{\mathcal{A}}$. Then, by Lemma $3.5(1)$ of [6] we get that $f_{t}^{(i)} \longrightarrow g_{t} \forall t \in[0,+\infty]$ in the strong operator topology in $\mathcal{A}$. Hence $f_{t}^{(i)} \longrightarrow g_{t} \forall t \in[0,+\infty]$ in the weak operator topology in $\mathcal{A}$. This implies that $\varphi\left(f_{t}^{(i)}\right) \longrightarrow \varphi\left(g_{t}\right)$ $\forall t \in[0,+\infty]$. Note that

$$
\mathcal{S}_{\theta}^{\sigma}\left(f_{i}\right)=\mathcal{M}_{0} f_{t_{0}}^{(i)}+\sum_{j=1}^{n} \varphi\left(f_{t_{j}}^{(i)}\right)\left(\mathcal{M}_{\tau_{j}}-\mathcal{M}_{\tau_{j-1}}\right),
$$

and

$$
\varphi\left(f_{t_{j}}^{(i)}\right)\left(\mathcal{M}_{\tau_{j}}-\mathcal{M}_{\tau_{j-1}}\right) \longrightarrow \varphi\left(g_{t_{j}}\right)\left(\mathcal{M}_{\tau_{j}}-\mathcal{M}_{\tau_{j-1}}\right) \quad \text { for } j=1,2, \ldots, n .
$$

Since the strong operator topology is continuous under multiplication, we have

Consequently, we get

$$
\mathcal{M}_{0}(\cdot) f_{t_{0}}^{(i)} \longrightarrow \mathcal{M}_{0}(\cdot) g_{t_{0}}
$$

$$
\mathcal{S}_{\theta}^{\sigma}\left(f_{i}\right) \longrightarrow \mathcal{S}_{\theta}^{\sigma}(g)
$$

in the strong-operator topology, which finishes the proof.

Acknowledgement. I would like to thank the referee for many valuable comments and remarks which substantially improved the paper. 


\section{References}

[1] Barnett, C., Supermartingales on semifinite von Neumann algebras, J. London Math. Soc. (2) 24 (1981), 175-181.

[2] Barnett, C., Camillo, V., Stopping and projections of non-adapted processes, Soochow J. Math. 23 (1997), 187-212.

[3] Barnett, C., Goldstein, S., Wilde, I. F., Quantum stopping times and Doob-Meyer decompositions, J. Operator Theory 35 (1996), 85-106.

[4] Barnett, C., Thakrar, B., Time projection in a von Neumann algebra, J. Operator Theory 18 (1987), 19-31.

[5] Barnett, C., Thakrar, B., A non-commutative random stopping theorem, J. Funct. Anal. 88 (1990), 342-350.

[6] Barnett, C., Volitis, S., Stopping and integration in a product structure, J. Operator Theory 34 (1995), 145-175.

[7] Barnett, C., Wilde, I. F., Random times and time projections, Proc. Amer. Math. Soc. 110 (1990), 425-440.

[8] Barnett, C., Wilde, I. F., Random times, predictable processes, and stochastic integration in finite von Neumann algebras, Proc. London Math. Soc. (3) 67 (1993), $355-383$.

[9] Dixmier, J., Von Neumann Algebras, North-Holland Math. Library 27, NorthHolland Publishing Co., Amsterdam-New York, 1981.

[10] Mohammed, A. A. A., Stochastic integration in finite von Neumann algebras, Ph.D. Thesis, Faculty of Mathematics, Łódź University, Łódź, 2000.

[11] Yeadon, F. J., Non-commutative $L^{p}$-spaces, Math. Proc. Cambridge Philos. Soc. 77 (1975), 91-102.

Abdulrahman A. A. Mohammed

BAGHDAD UNIVERSITY

College OF SCIENCE

DEPARTMENT OF MATHEMATiCS

BAGHDAD, IRAQ

E-MAIL: ABDULRAHMAN62@YAHOO.COM 